REVISTA DE LA CEPAL 82 A ABIL 2004

\title{
La sustitución de importaciones en las industrias de alta tecnología:
}

\section{Prebisch renace en Asia}

\author{
Alice H. Amsden
}

$\mathrm{P}$

uede decirse que Prebisch renace en Asia en la medida en que los gobiernos de las principales economías de este continente siguen fomentando activamente la sustitución de importaciones de piezas y componentes para su utilización en la alta tecnología. Pero las medidas de promoción que se aplican con tal fin trascienden la protección arancelaria y, a juzgar por el nivel de desempeño, han resultado muy eficaces. En la actualidad, América Latina está rezagada con respecto a los países asiáticos de industrialización tardía porque perdió la oportunidad de participar directamente en la revolución de la tecnología de la información. Pero la región todavía puede inspirarse en las economías de Asia, en vez de en los principios del Consenso de Washington, para desarrollar un sector maduro de alta tecnología, más allá del campo de la electrónica, 


\section{I}

\section{Introducción}

En la década de 1950 comenzó a asociarse la figura de Raúl Prebisch con las controvertidas políticas latinoamericanas de sustitución de importaciones y contrarias al crecimiento exportador. ${ }^{1}$ Pese a la positiva evolución de la industria básica como consecuencia de la sustitución de importaciones, hacia mediados del decenio de 1980, e incluso antes, estas políticas fueron criticadas por su ineficiencia y por los problemas de balanza de pagos que planteaban. Con el auge mundial de la liberalización, las políticas e instituciones que la habían apoyado fueron desmanteladas. América Latina abrió sus mercados a las exportaciones de ultramar y a mayores inversiones de las empresas transnacionales.

Paradójicamente, mientras América Latina iba borrando su pasado, Asia reinventaba la sustitución de importaciones para las industrias de alta tecnología, y esta región crecía más que América Latina con un régimen liberal. Al principio se importaba una alta proporción del valor de la mayoría de los aparatos electrónicos (por ejemplo, computadoras portátiles y teléfonos celulares). La política gubernamental se orientaba hacia la sustitución de importaciones de las piezas y componentes utilizados en la fabricación de esos productos con el fin de crear empleos bien pagados en el mercado interno, y empresas de capital nacional capaces de competir en el mercado externo. Las políticas de sustitución de importaciones para fomentar la producción de alta tecnología en China, India, la República de Corea y la provincia china de Taiwán (en adelante se usará el término "Asia" para referirse a estas cuatro economías) generaban menos distorsiones que las utilizadas para promover la industria de tecnología media, en la medida en que no suponían una alta protección arancelaria. Sin embargo, los países asiáticos comenzaron a desempeñar un papel muy activo en el despegue del sector de alta tecnología, papel que

Este trabajo fue preparado originalmente para el seminario "La teoría del desarrollo en los albores del siglo XXI", organizado por la CEPAL en Santiago de Chile para conmemorar el centenario del nacimiento de Raúl Prebisch.

${ }^{1}$ Esto último es injusto, ya que en 1968 Prebisch recomendaba con vehemencia al gobierno argentino que combinara la sustitución de las importaciones con la actividad exportadora (Mallon y Sourrouille, 1975) le habría resultado familiar a Prebisch. La participación gubernamental en Asia (es decir, en las cuatro economías mencionadas) llegaba a los más ínfimos detalles, tales como seleccionar una a una las compañías beneficiarias de subsidios (que se traducían, por ejemplo, en su instalación en un parque científico) y desprenderse de ciertas secciones de los laboratorios gubernamentales para crear nuevas empresas.

El fomento de la ciencia y la tecnología y del desarrollo regional en Asia se apartaba de los cánones del mercado libre incorporados en el Consenso de Washington, pero esas medidas intervencionistas eran consideradas legítimas por la Organización Mundial del Comercio (OMC). Los miembros de la omc todavía pueden promover la ciencia y la tecnología en el nivel empresarial, como se hace en los Estados Unidos a través de su Departamento de Defensa (gran aliado de la industria de las computadoras) y su Instituto Nacional de Salud (adalid de la biotecnología). Asimismo, pueden fomentar el desarrollo regional (mediante subsidios para las compañías que se ubiquen en zonas de bajo desarrollo), como tienden a hacer los países de la Unión Europea ante desigualdades de ingreso entre norte y sur.

En las primeras secciones de este trabajo se intenta evaluar el alcance de la intervención estatal en la industria de alta tecnología a partir del caso de la provincia china de Taiwán. ${ }^{2}$ El análisis puede interesar a los economistas que se preguntan cómo podrían reanimarse las exangües industrias de alta tecnología de Argentina, de Brasil (algo menos exangües), de Chile y de México. ${ }^{3}$ En la última sección se examinan las razones del estancamiento de las industrias de esta índole en América Latina y se proponen medidas para implantar y desarrollar una estrategia similar a la asiática. Se pone el acento en la empresa nacional y no en la de propiedad extranjera.

\footnotetext{
${ }^{2}$ Estas secciones se basan en Amsden y Chu (2003).

${ }^{3}$ En adelante se utilizará el término "América Latina" para referirse a estos cuatro países.
} 


\section{II}

\section{La propiedad nacional}

\section{y la industria de alta tecnología}

El establecimiento de industrias de alta tecnología en países de industrialización reciente —incluso de industrias maduras que constituyen la mayor parte de las de alta tecnología en esos países - requiere capacidad empresarial, en cuanto implica la creación de los recursos humanos especializados de los cuales dependen esas industrias. Existe un alto riesgo de que estas personas abandonen la empresa que les dio capacitación. Por lo tanto, o bien el gobierno emprende inicialmente la tarea de formar especialistas, con el objetivo de difundir estas capacidades al sector privado, o bien la empresa privada debe ser lo suficientemente emprendedora como para crecer y diversificarse al grado de poder retener los recursos humanos en que ha invertido.

En los países de industrialización reciente, todavía en el año 2000 la industria de alta tecnología apuntaba a fabricar productos maduros. Así, por ejemplo, en la República de Corea y en la provincia china de Taiwán la producción de computadoras portátiles y teléfonos celulares comenzó cuando los países de salarios altos llevaban varios años produciéndolos a gran escala. Los productos maduros suponen un margen de utilidad bruta que disminuye rápidamente conforme pasa el tiempo. Para obtener beneficios de ellos, la empresa debe iniciar la producción con rapidez, antes de que la rentabilidad caiga aún más, y hacerlo a gran escala para superar los exiguos márgenes. Este salto, junto con la adaptación de los productos para llegar lo antes posible al mercado, conlleva riesgos y exige iniciativa empresarial, puesto que obliga a invertir en una nueva serie de capacidades laborales, coordinar su formación, conseguir los fondos para su financiamiento, ponerlas en práctica y vigilar su evolución (Amsden y Chu, 2003).

Esas capacidades imprescindibles pueden fomentarse desde organizaciones estatales o privadas, pero las que tienen el mayor incentivo para liderar este proceso son las empresas de capital nacional. En comparación con las empresas extranjeras, las nacionales tienen costos de oportunidad más bajos, mejor conocimiento del terreno y más probabilidades de aprovechar activos inmovilizados en industrias locales relacionadas. Por lo tanto, las primeras que ingresen a la industria de alta tecnología en los países de industrialización reciente serán, probablemente, entidades de capital nacional (estatales o privadas). Es posible que las empresas transnacionales hayan tenido en algún momento, en los países de industrialización reciente, un papel formativo en la creación de industrias con uso intensivo de mano de obra y para mercados de exportación, y es posible también que ingresen al campo de las industrias de alta tecnología y aceleren el impulso de crecimiento existente. ${ }^{4}$ Sin embargo, cabe suponer que los pioneros de la industria de alta tecnología en esos países serán las empresas de propiedad nacional con experiencia directa o indirecta en los sectores de tecnología media u otro tipo de actividad económica, como la comercialización de productos extranjeros (Amsden e Hikino, 1994).

En el cuadro 1 se muestra la participación de la industria de alta tecnología en el valor agregado industrial de algunos países latinoamericanos y asiáticos en 1980 y en 1995, último año para el cual se dispone estadísticas comparables. Es notable cuanto más elevada es esa participación en China, India, la República de Corea y la provincia china de Taiwán, que en Argentina, Chile y México. Sólo Brasil tiene una proporción de alta tecnología en su sector manufacturero comparable a la de los países asiáticos.

Estas diferencias pueden atribuirse al origen del capital de las empresas y a las iniciativas gubernamentales. Por otra parte, la proporción de empresas con capital nacional o extranjero y el grado de intervención del Estado tienen profundas raíces históricas relacionadas con la naturaleza de la experiencia manufacturera y la transferencia de tecnología en la preguerra (Amsden, 2001). ${ }^{5}$ Pueden distinguirse dos

\footnotetext{
${ }^{4}$ En Amsden (2001) se analiza la secuencia histórica de la inversión extranjera directa.

${ }^{5}$ No se estudian los casos de Malasia, Indonesia y Tailandia, cuyos sectores manufactureros son relativamente nuevos en comparación con aquellos de los cuatro países asiáticos y los cuatro latinoamericanos que se consideran aquí. La participación del sector de alta tecnología en el valor agregado de la industria manufacturera en Malasia se debe al ensamblado de elementos electrónicos, que hace uso intensivo de mano de obra y no es propiamente una industria de alta tecnología en cuanto a las capacidades laborales requeridas. Este tema se aborda
} 


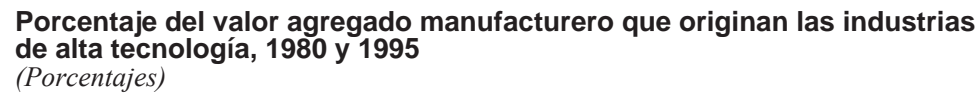

\begin{tabular}{|c|c|c|c|c|c|c|c|c|c|}
\hline 1995 & Argentina & Brasil & Chile & China & India & Indonesia & $\begin{array}{l}\text { República } \\
\text { de Corea }\end{array}$ & Malasia & México \\
\hline Otros productos químicos & 3,5 & 10,1 & 8,0 & 1,9 & 7,9 & 3,6 & 4,7 & 2,2 & 7,2 \\
\hline Maquinaria no eléctrica & 3,1 & 7,5 & 1,8 & 11,1 & 8,3 & 1,0 & 8,4 & 5,0 & 3,3 \\
\hline Maquinaria eléctrica & 3,0 & 8,0 & 1,5 & 9,9 & 8,4 & 3,1 & 14,4 & 27,4 & 3,2 \\
\hline Equipo de transporte & 7,4 & 10,4 & 2,0 & 6,3 & 8,5 & 8,9 & 10,7 & 4,7 & 10,1 \\
\hline Bienes científicos y profesionales & 0,4 & 0,8 & 0,2 & 1,1 & 0,7 & 0,1 & 0,8 & 1,2 & 1,7 \\
\hline \multirow[t]{2}{*}{ Total } & 17,4 & 36,8 & 13,3 & 30,2 & 33,7 & 16,6 & 39,1 & 40,5 & 25,6 \\
\hline & $\begin{array}{l}\text { Provincia } \\
\text { china de } \\
\text { Taiwán }\end{array}$ & Tailandia & Turquía & $\begin{array}{l}\text { Resto, } \\
\text { promedio }\end{array}$ & & Japón & Francia & $\begin{array}{l}\text { Reino } \\
\text { Unido }\end{array}$ & $\begin{array}{l}\text { Estados } \\
\text { Unidos }\end{array}$ \\
\hline Otros productos químicos & 2,7 & 2,5 & 4,7 & 4,9 & & 5,8 & 6,1 & 7,0 & 6,8 \\
\hline Maquinaria no eléctrica & 5,2 & 3,3 & 4,5 & 5,2 & & 12,1 & 7,0 & 11,3 & 10,5 \\
\hline Maquinaria eléctrica & 17,3 & 5,5 & 6,0 & 9,0 & & 14,7 & 10,0 & 8,4 & 9,6 \\
\hline Equipo de transporte & 7,4 & 5,2 & 6,7 & 7,4 & & 10,6 & 10,9 & 10,4 & 11,6 \\
\hline Bienes científicos y profesionales & 1,0 & 0,9 & 0,3 & 0,8 & & 1,3 & 1,5 & 1,6 & 5,8 \\
\hline Total & 33,6 & 17,3 & 22,3 & 27,2 & & 44,4 & 35,6 & 38,8 & 44,3 \\
\hline 1980 & Argentina & Brasil & Chile & China & India & Indonesia & $\begin{array}{l}\text { República } \\
\text { de Corea }\end{array}$ & Malasia & México \\
\hline Otros productos químicos & 4,9 & 4,9 & 6,5 & 3,3 & 8,1 & 7,1 & 5,2 & 3,2 & 5,2 \\
\hline Maquinaria no eléctrica & 5,5 & 10,0 & 1,9 & 15,1 & 8,6 & 1,6 & 3,4 & 3,2 & 4,8 \\
\hline Maquinaria eléctrica & 3,7 & 6,3 & 1,8 & 3,6 & 8,1 & 5,3 & 8,1 & 12,3 & 4,4 \\
\hline Equipo de transporte & 9,3 & 7,8 & 2,5 & 3,4 & 8,3 & 6,4 & 5,9 & 4,2 & 6,9 \\
\hline Bienes científicos y profesionales & 0,4 & 0,6 & 0,1 & 9,2 & 0,7 & 0,1 & 1,1 & 0,7 & 0,7 \\
\hline \multirow[t]{2}{*}{ Total } & 23,8 & 29,8 & 12,9 & 34,6 & 33,9 & 20,4 & 23,8 & 23,6 & 22,1 \\
\hline & $\begin{array}{l}\text { Provincia } \\
\text { china de } \\
\text { Taiwán }\end{array}$ & Tailandia & Turquía & $\begin{array}{l}\text { Resto, } \\
\text { promedio }\end{array}$ & & Japón & Francia & $\begin{array}{l}\text { Reino } \\
\text { Unido }\end{array}$ & $\begin{array}{l}\text { Estados } \\
\text { Unidos }\end{array}$ \\
\hline Otros productos químicos & 1,0 & 2,7 & 3,6 & 4,7 & & 4,6 & 3,9 & 4,6 & 4,6 \\
\hline Equipo de transporte & 2,5 & 3,7 & 5,0 & 5,5 & & 9,5 & 11,0 & 10,7 & 10,6 \\
\hline Bienes científicos y profesionales & 0,9 & 0,3 & 0,1 & 1,2 & & 1,7 & 1,4 & 1,3 & 3,6 \\
\hline Total & 13,4 & 12,4 & 17,6 & 22,3 & & 38,7 & 35,2 & 38,9 & 41,9 \\
\hline
\end{tabular}

Fuente: Amsden (2001).

tipos principales de transferencia: por migración y por colonización. La importancia atribuida al capital nacional y a las iniciativas gubernamentales en el fomento de las industrias de alta tecnología tiende a ser mayor en países con experiencias de tipo colonial que en aquellos con experiencias de tipo migratorio en la época de preguerra. ${ }^{6}$

con más detalle en Amsden (2001). Sin embargo, tanto Tailandia como Malasia han comenzado a invertir cantidades considerables en actividades de investigación y desarrollo. La inversión en tecnología de Tailandia nunca fue insignificante. Hace más de 25 años Indonesia envió a un nutrido grupo de ingenieros al Departamento de Aeronáutica y Astronáutica del MIT, para construir con ellos su industria aeroespacial.

${ }^{6} \mathrm{Al}$ estudiar varios países, la cuantificación de la propiedad extranjera
En las experiencias de tipo migratorio, la capacidad tecnológica y la organización empresarial se transfirieron a través de personas (y más tarde, empresas) que emigraron e iniciaron sus operaciones en entornos de industrialización reciente. Los estadounidenses y europeos emigrados a América Latina y los chinos en Asia (Malasia, Indonesia y Tailandia) han sido una

frente a la nacional en cualquier industria, o en el sector manufacturero en su conjunto, tropieza con diferentes definiciones de la empresa foránea respecto al porcentaje del capital que está en poder de extranjeros. Sin embargo, los datos disponibles para varios países sugieren que la inversión extranjera directa es mucho más importante en el sector manufacturero de América Latina que en el de Asia (véase Amsden, 2001). 
fuente importante de conocimientos técnicos y comerciales modernos, y de formación de nuevas capacidades. Los límites entre la propiedad extranjera y la nacional se han ido desdibujando y la política gubernamental es, en el mejor de los casos, ambivalente en lo que toca a promover esta última por sobre la extranjera. Las políticas de identidad no dejan en claro qué es extranjero y qué es nacional.

En la experiencia industrial de tipo colonial anterior a la segunda guerra mundial, las técnicas empresariales y las capacidades que llegaban del extranjero eran transferidas por compañías cuya sede estaba en la metrópoli colonial. Por ejemplo, entre las empresas extranjeras de la India predominaban las británicas; en la República de Corea y la provincia china de Taiwán, las japonesas. Esta forma de transferencia de tecnología favoreció a las empresas de capital nacional, ya que el colonialismo acabó en descolonización, lo que tuvo muchos efectos positivos en los países que habían logrado adquirir experiencia industrial en la preguerra. El dominio político extranjero fue reemplazado por el gobierno político nacional y, en general, las empresas coloniales pasaron a manos nacionales. En Indonesia, unas 400 compañías de propiedad neerlandesa quedaron en el país (prácticamente destruidas) cuando terminó el dominio colonial (Lindblad, 1996). En la India, las empresas británicas se vendieron a compradores indios o no pudieron resistir la competencia con ellos (Tomlinson, 1981). En la República de Corea y la provincia china de Taiwán, las empresas manufactureras y los bancos japoneses quedaron en manos de gobiernos nacionales (Amsden, 1989; Fields, 1995). En China, las empresas extranjeras fueron expropiadas después de la revolución comunista. La descolonización allanó así el terreno para el crecimiento de las empresas de propiedad nacional, mientras que inversores externos tendieron a desplazar a las empresas de capital nacional en sectores con economías de escala. ${ }^{7}$ Por su parte, las empresas de propiedad nacional, sobre todo las que provenían de industrias de tecnología media, a menudo fueron las que estuvieron detrás del auge de industrias y servicios de alta tecnología (de computadoras, telecomunicaciones, finanzas y trenes de alta velocidad), como ocurrió en la República de Corea y en la provincia china de Taiwán. ${ }^{8}$

La descolonización también fomentó una cultura de nacionalismo que dio pie a demandas de reforma

\footnotetext{
${ }^{7}$ El caso de la industria argentina del automóvil se describe en Cochran y Reina (1962).

8 Sobre este último caso, véase Amsden y Chu (2003).
}

agraria y a políticas públicas encaminadas a fomentar las empresas de propiedad nacional y las industrias de alta tecnología. El nacionalismo económico asumió muchas formas, pero todas ellas apuntaban a crear una alternativa a la empresa transnacional y a generar los medios para ascender por la vía de las ventajas comparativas, superando la etapa de las industrias con uso intensivo de mano de obra o materias primas. Para conseguirlo era preciso, ante todo, establecer laboratorios de investigación y desarrollo de propiedad gubernamental, como los que crearon China e India con fines de defensa y que luego se convirtieron en semilleros para las industrias privadas de alta tecnología. En segundo lugar, se necesitaban inversiones en la educación superior, que eran más bien elevadas en Asia.

Por último, el nacionalismo tendió a fomentar un espíritu de igualdad, que a todas luces faltaba bajo el régimen colonial. Los cuatro países asiáticos analizados implantaron la reforma agraria con mayor o menor intensidad (la reforma más radical fue la de China, la menos radical la de India). Gracias a la reforma agraria se abolieron los latifundios que obtenían rentas ricardianas (es decir, superiores a las utilidades normales) en la época anterior a la segunda guerra mundial. Al desaparecer esas posibilidades de lucro, los costos de oportunidad de invertir en la manufactura bajaron, con lo cual la inversión en el sector industrial se convirtió en una opción financiera más atractiva que la que ofrecían las tenencias consolidadas de tierras y derechos mineros, típicas del sector agrícola en su acepción más amplia. La reducción de los costos de oportunidad fuera del sector manufacturero también hizo menos oneroros para el gobierno los subsidios a la industria destinados a atraer capitales y recursos humanos desde la agricultura hacia la actividad manufacturera y crear nuevas industrias basadas en la sustitución de importaciones (Amsden, 2001).

Por lo tanto, como se dijo en la sección anterior, la actividad industrial de alta tecnología, medida en el cuadro 1 como la participación de la industria de alta tecnología en el valor agregado industrial total, es más elevada en Asia que en América Latina. La excepción es Brasil, donde el Estado emprendió un proyecto de carácter muy nacionalista para desarrollar la capacidad local de fabricar minicomputadoras. El proyecto en su conjunto fracasó, en parte por deficiencias de la educación superior y en parte porque las empresas de capital nacional tenían mucha más experiencia en el ámbito de las finanzas que en el de la manufactura (Evans, 1995; Sridharan,1996).

Así pues, la diferente evolución de las industrias 
de alta tecnología asiáticas y latinoamericanas puede atribuirse a factores históricos: concretamente, a la forma (por migración o por colonización) en que adquirieron experiencia manufacturera con anterioridad a la segunda guerra mundial.

Veamos ahora cómo y por qué se debe fomentar la industria de alta tecnología en economías dominadas por emigrantes. Sin embargo, se examinará primero el caso de la provincia china de Taiwán, donde tanto la inmigración (procedente en este caso de la región de Shanghai, China) como el proceso de descolonización fueron cruciales para superar la etapa de desarrollo tardío, basado en industrias de tecnología media.

\section{III}

\section{El liderazgo gubernamental en las industrias de alta tecnología de la provincia china de Taiwán}

El papel del gobierno taiwanés en la evolución de la industria de alta tecnología fue de suma importancia, ya que tenía por objeto crear nuevos segmentos de mercado en que pudieran competir las empresas nacionales. Su estrategia contemplaba la sustitución de importaciones y la generación de proveedores de componentes en torno a una empresa guía (es decir, la primera empresa, entre las recién llegadas, en ingresar a una industria madura de alta tecnología). Mientras que el gobierno había creado nuevas industrias en la antigua economía aprovechando las empresas estatales y los instrumentos de la política de sustitución de importaciones -como la protección arancelaria, las exigencias de contenido nacional y los bancos de desarrollo-, en la nueva economía se sirvió de los subproductos de los institutos de investigación fiscales y los parques científicos, y de herramientas de la política de sustitución de importaciones (como subsidios para las actividades públicas y privadas de investigación científica y tecnológica, exenciones tributarias y condiciones financieras favorables para los que se establecieran en los parques científicos).

Hacia el año 2000 había más de 15.000 profesionales en la provincia china de Taiwán que habían trabajado alguna vez con el Instituto de Investigación de Tecnología Industrial (ITRI). ${ }^{9}$ De ellos, más de 12.000 se habían incorporado a alguna industria de ese tipo. De estos últimos, unos 5.000 habían

\footnotetext{
${ }^{9}$ Industrial Technology Research Institute, principal centro de investigación del gobierno dedicado a la industria de alta tecnología.
}

trabajado en el parque científico industrial de Hsinchu. ${ }^{10}$ El ITRI fue también el origen de los dos pilares de la industria de semiconductores de Taiwán: United Microelectronics Corporation (UMC) y Taiwan Semiconductor Manufacturing Company (TSMC).

El gobierno tenía el propósito de despejar los estrangulamientos tecnológicos para que las industrias nacionales que ingresaban tarde a un sector pudieran competir a nivel internacional en las nuevas subindustrias de alta tecnología y traspasar sus conocimientos técnicos a los proveedores locales de piezas y componentes. En la década de 1970, el acelerado crecimiento de las exportaciones con alto contenido de mano de obra había agotado las reservas laborales locales "ilimitadas". Se estaban ejecutando grandes proyectos para la industria pesada. Por lo tanto, era a todas luces evidente que había que crear nuevas oportunidades de crecimiento basadas en la industria de alta tecnología y que el gobierno debía tener un papel de primer orden en su creación. 'Para muchos dirigentes taiwaneses, el mecanismo clásico de asignación de recursos mediante los precios resultaba demasiado lento para promover el desarrollo industrial. Abogaban por la aplicación de medidas más directas de política industrial para acelerar el desarrollo de la industria de alta tecnología'. ${ }^{11}$

El gobierno estimulaba la industria de alta tecnología en varios frentes: por medio de la política

\footnotetext{
${ }^{10}$ Los informes ITRI (2000a y 2000b) e ITIS (1989) se encuentran en http://www.itri.org.tw/eng/about/annual/annua198/spec_pg3.jsp 11 San (1995, p. 35). En este trabajo, las citas que aparecen entre comillas simples han sido traducidas del inglés.
} 
fiscal, la creación de parques científicos y las inversiones dinámicas de las instituciones gubernamentales de investigación y desarrollo, algunas de las cuales cumplían múltiples objetivos. El ITRI, por ejemplo, emprendió proyectos clave de tecnología para dar un empuje inicial a industrias esenciales, como la de semiconductores y la de computadoras personales; fue así como los derivados de estas industrias se convirtieron en las principales empresas fabricantes de circuitos integrados. Además, participó activamente en proyectos para explorar los campos principales en que se creía que el sector privado tendría posibilidades lucrativas de inversión futura. Luego de la puesta en marcha de una industria, el ITRI iniciaba proyectos más pequeños para sustituir los componentes clave. El objetivo del gobierno era crear oportunidades de crecimiento y valor agregado locales, además de mejorar el nivel de la tecnología local. Todos los tipos de promoción convergían en las industrias calificadas de "estratégicas" en función de la densidad de uso de tecnología, el valor agregado, el mercado potencial, los encadenamientos industriales, el consumo de energía y el componente de contaminación.
En 1992, el gobierno aprobó la ley de desarrollo de componentes y productos críticos, en la que se seleccionan 66 insumos para la sustitución de importaciones a fin de reducir el persistente déficit comercial con Japón. ${ }^{12}$ Aunque los usuarios taiwaneses preferían los productos importados cuando se trataba de componentes de alta tecnología, la escasez de esos componentes hacía prever precios altos y grandes beneficios para las empresas que pudieran fabricarlos en vez de importarlos. Los usuarios de estos insumos tenían el incentivo adicional de fabricarlos internamente a fin de estabilizar sus suministros. Por su parte, el gobierno se comprometió a sustituir los componentes de alta tecnología para evitar que las labores de manufactura se trasladaran al exterior.

El papel protagónico del gobierno en el fortalecimiento de las actividades de ciencia y tecnología se ilustra a continuación mediante los ejemplos de los discos compactos, las pantallas de cristal líquido y los circuitos integrados.

\section{IV}

\section{Sustitución de importaciones con promoción de alta tecnología}

\section{CD-ROM}

El CD-ROM (dispositivo de almacenamiento óptico de datos) ${ }^{13}$, fue elegido por el gobierno taiwanés como objetivo industrial en 1992, luego de amplias discusiones entre funcionarios de gobierno, académicos y destacados empresarios. También se identificaron varias tecnologías

\footnotetext{
${ }^{12}$ El déficit comercial anual de la provincia china de Taiwán con Japón aumentó hasta los 10.000 millones de dólares en 1991, en comparación con los 2.000 a 3.000 millones registrados en el quinquenio 1980-1984. Japón era el único socio comercial con el cual Taiwán mantenía permanentemente un gran déficit comercial. Sin embargo, la participación de las importaciones taiwanesas desde Japón en el total se mantuvo relativamente estable, en torno al 30\%. Por lo tanto, la dependencia de las importaciones de Japón era más bien un indicador de dependencia tecnológica y no de desequilibrio comercial. En este sentido, podría afirmarse que la ley de desarrollo de productos y componentes críticos tuvo menos que ver con la estructura del comercio que con un mejoramiento industrial.
}

clave que serían promovidas, como la cabeza de lectura óptica de discos. El Departamento de tecnología industrial del Ministerio de Economía se encargó del denominado "lado de la oferta": instó a las instituciones de investigación, principalmente al ITRI, a presentar propuestas de investigación y desarrollo a fin de diseñar los artículos seleccionados. Los recursos provinieron del Fondo de ciencia y tecnología en cuatro años consecutivos, de 1993 a 1996. A fines de este período el presupuesto total rondaba los 10 millones de dólares.

La Junta de desarrollo industrial del Ministerio gestionó el lado de la demanda: invitó a empresas privadas (sobre la base de criterios específicos) a

\footnotetext{
${ }^{13}$ La información sobre los lectores de CD-ROM se deriva de las fuentes siguientes: Industrial Development Bureau (varios años) ITRI (1997), ERSO (1994), Hsiao (1994), y Market Intelligence Center (varios años).
} 
participar en el proceso de desarrollo. Los programas eran dos: uno sobre la reglamentación para el desarrollo de nuevos productos industriales por la empresa privada y otro acerca de la reglamentación sobre la asistencia gubernamental para el desarrollo de productos principales. Ambos programas otorgaban asistencia para investigación y desarrollo a las empresas privadas que se dedicaran a idear nuevos productos. Los préstamos debían reembolsarse cuando se concretaran las ventas.

El proyecto de CD-ROM involucró a 25 empresas en un proceso integrado de desarrollo y transferencias de tecnología. ${ }^{14}$ Se derivaron cuatro patentes para los equipos de CD-ROM y 24 para las cabezas lectoras. El despegue fue sorprendentemente rápido. Como se ve en el cuadro 2, la participación de la provincia china
CUADRO 2

Fabricación de CD-ROM, 1991-1999a

\begin{tabular}{lcccc}
\hline Año & \multicolumn{2}{c}{$\begin{array}{c}\text { Producción, } \\
\text { miles de unidades }\end{array}$} & B/A & $\begin{array}{c}\text { Cooperación } \\
\text { del ITRI }\end{array}$ \\
\cline { 2 - 3 } & A. Mundial & B. De Taiwán & $(\%)$ & $\left(\mathrm{N}^{\mathbf{o}}\right.$ de empresas $)$ \\
\hline 1991 & 936 & & & 1 \\
1992 & 1050 & & & 7 \\
1993 & 6740 & & & 25 \\
1994 & 17966 & 218 & 1 & 25 \\
1995 & 38572 & 3600 & 9 & 25 \\
1996 & 51000 & 9170 & 18 & 25 \\
1997 & 61000 & 16000 & 26 & \\
1998 & 89300 & 30780 & 35 & \\
1999 & 96860 & 48690 & 50 & \\
\hline
\end{tabular}

Fuente: ITRI (1997) y Market Intelligence Center (varios años).

a CD-ROM: disco compacto con memoria sólo de lectura.

CUADRO 3

Avance tecnológico en la industria del CD-ROM, 1994-1999a

(Porcentajes de la producción total)

\begin{tabular}{|c|c|c|c|c|c|c|c|c|c|c|c|c|c|c|}
\hline \multirow[t]{2}{*}{ Año } & \multicolumn{13}{|c|}{ Velocidad } & \multirow[b]{2}{*}{ Total } \\
\hline & 2 & 4 & 6 & 8 & $10-12$ & 16 & 20 & 24 & 32 & 36 & 40 & $44-48$ & $>50$ & \\
\hline $\begin{array}{l}1994 \\
1995 \\
1996 \\
1997^{b} \\
1998^{c} \\
1999^{c}\end{array}$ & $\begin{array}{r}100 \\
40\end{array}$ & 47 & $\begin{array}{l}13 \\
13\end{array}$ & 67 & 20 & 23 & 22 & $\begin{array}{r}55 \\
2\end{array}$ & 11 & $\begin{array}{r}27 \\
6\end{array}$ & $\begin{array}{l}60 \\
31\end{array}$ & 47 & 16 & $\begin{array}{l}100 \\
100 \\
100 \\
100 \\
100 \\
100\end{array}$ \\
\hline
\end{tabular}

Fuente: Market Intelligence Center (varios años).

a CD-ROM: disco compacto con memoria sólo de lectura.

b Segundo semestre.

c Cuarto trimestre.

de Taiwán en la producción mundial de $\mathrm{CD}$-ROM pasó de $1 \%$ en 1994 (218.000 unidades) a 50\% cinco años después (48.690.000 unidades).

Aunque las empresas que adquirieron esta tecnología del ITRI pudieron comenzar de inmediato las operaciones de ensamblado, y aunque a la sazón el producto estaba en su etapa de madurez, el proceso de innovación tecnológica siguió siendo muy rápido. Como se indica en el cuadro 3 , los fabricantes tenían que renovar sus conocimientos técnicos constantemente a fin de producir CD-ROM de mayor velocidad. Además, tenían que importar componentes clave de Japón.

\footnotetext{
${ }^{14}$ Entre las empresas participantes entrevistadas figuraban BTC, Inventec, Acer, U-Max y Lite-On. Las empresas BTC y Lite-On también participaron en el proyecto para desarrollar la cabeza lectora de CD-ROM.
}

Paulatinamente, comenzó la producción local de discos y soportes multidisco, pero los dos insumos más importantes, es decir la cabeza lectora óptica y el circuito integrado específico (ASIC, según sus siglas en inglés), todavía se importaban después de 1996, aunque el ITRI estaba en vías de desarrollarlos.

Las empresas taiwanesas adelantaron a las japonesas como grandes productoras de CD-ROM, pero las compañías japonesas estaban adoptando modelos nuevos y mejorados, como el DVD-ROM y el CD-RW. En general, las firmas taiwanesas eran reacias a entrar en la producción de DVD-ROM, porque consideraban que las regalías exigidas por las empresas japonesas eran prohibitivas. Sin embargo, el ITRI transfirió la tecnología del DVD-ROM a 13 firmas en 1997. Hacia el año 2000 el precio del DVD-ROM era dos veces superior al del CD-ROM, 
y se esperaba que el reemplazo de uno por otro fuera un proceso lento.

\section{Pantallas de cristal líquido}

Las pantallas de cristal líquido (LCD) ${ }^{15}$ aparecieron a fines del decenio de 1970 y durante el de 1980, inventadas por firmas japonesas, al comienzo en sus formas más simples - es decir, nemático torsionado (TN) y nemático supertorsionado (STN) - y luego en su forma compleja de transistor de película delgada (TFT). ${ }^{16}$ Ambos productos representaban un gran reto de fabricación por sus grandes exigencias tanto financieras como de manufactura. La rentabilidad dependía de una baja tasa de piezas defectuosas y un alto rendimiento.

Hacia mediados de la década de 1990, algunos chaebol coreanos (como Samsung, Hyundai y LG), en colaboración con los ministerios encargados de promover la innovación tecnológica, habían logrado penetrar en la industria y presentar cierto desafío a la hegemonía japonesa. Algunas empresas taiwanesas ya estaban produciendo pantallas TN y STN a comienzos de esa década, pero dudaban en ingresar al mercado de las pantallas TFT, de mucho mayor densidad de capital.

Dos circunstancias pudieron impulsarlas a actuar. Antes de la crisis financiera asiática de 1997, Samsung, Hyundai y LG habían programado una gran expansión para ponerse al nivel de los fabricantes japoneses, pero la crisis los obligó a postergar sus planes. Por su parte, los conglomerados japoneses, que sufrían las consecuencias de una crisis prolongada y de un exceso de capacidad instalada, no pudieron o no quisieron seguir efectuando las grandes inversiones que se requerían para mantener la competitividad en el mercado de pantallas TFT. Así, sólo unos pocos decidieron cooperar con las empresas taiwanesas, concediéndoles licencias tecnológicas y encargándoles equipos de fabricación original (OEM, según sus siglas en inglés). ${ }^{17}$ Súbitamente, las principales empresas taiwanesas anunciaron sus planes para obtener tecnología de sus

\footnotetext{
15 La información sobre la fabricación de pantallas de cristal líquido proviene de Wong y Matthews (1998), Linden y otros (1998), e ITRI (1999 y 2000b).

${ }^{16}$ La pantalla de cristal líquido es el ejemplo más conocido del tipo de pantalla plana microelectrónica que se emplea en las calculadoras, las computadoras portátiles y otros aparatos.

${ }^{17}$ Una empresa conjunta de IBM y Toshiba fundada en 1989 dejó de fabricar pantallas de cristal líquido para computadoras en mayo de 2001. Toshiba aprovechará sus instalaciones en Japón para fabricar LCD para teléfonos celulares; IBM la empleará para productos de muy alta resolución, como los aparatos médicos (Nikkei Weekly, 2001).
}

asociados japoneses y realizar grandes inversiones para fabricar pantallas TFT.

La entrada de las firmas taiwanesas cambió la distribución mundial de la capacidad de producción de pantallas TFт. La participación taiwanesa en la capacidad mundial subió de cero en 1998 a $2 \%$ en 1999 , a $15 \%$ en 2000 , y a $26 \%$ en el primer trimestre de 2001 . Con estos enormes incrementos, el precio internacional de tales pantallas se desplomó. El precio de una pantalla TFT de 14,1 pulgadas para computadora portátil bajó de 1.100 dólares en el tercer trimestre de 1997 a poco más de 600 dólares en el cuarto trimestre del 2000. Con grandes inversiones y precios a la baja, las fusiones y las adquisiciones aparecieron en las agendas de las empresas.

El ITRI había iniciado un proyecto de desarrollo e investigación sobre pantallas TFT en 1988. Sin embargo, ninguna firma taiwanesa confió en la tecnología del ITRI cuando llegó el momento de invertir. En este aspecto, los esfuerzos del ITRI fueron un fracaso. Sin embargo, la alta capacidad de competencia de los grupos taiwaneses de alta tecnología que entraron en la producción de TFT dependía de la evolución tecnológica futura y se esperaba que el ITRI desempeñaría un papel protagónico en esa etapa ulterior. Este Instituto estableció el primer laboratorio de pantallas TFT con polisilicona de baja temperatura en 2000 y ha desarrollado algunos componentes clave para la fabricación de modelos de pantalla más avanzados.

\section{Diseño de circuitos integrados}

La base de la industria de semiconductores reticulados fue la creación de dos fábricas de categoría internacional por el gobierno de la provincia china de Taiwán: United Microelectronics Corporation (UMC) en 1980, que aún es de propiedad estatal, y Taiwán Semiconductor Manufacturing Company (TSMC) en 1987, de mayor tamaño. Ambas fueron subproductos de las fábricas experimentales de circuitos integrados creadas por la Organización de Investigación y Servicios para la Electrónica (ERSO), también propiedad del gobierno, aunque surgieron en épocas distintas y son fruto de proyectos diferentes. ${ }^{18} \mathrm{La}$ TSMC era también una "fundición" cuya única especialidad era la producción de laminados ultrafinos. No se interesó por la inversión en las operaciones auxiliares, a diferencia de los fabricantes de dispositivos integrados, que se

\footnotetext{
${ }^{18}$ La ERSO forma parte del ITRI
} 
organizaban verticalmente y dominaban la industria mundial de semiconductores. ${ }^{19}$ La estrategia de especialización fue resultado de una decisión deliberada del gobierno, en la que tuvieron influencia las opiniones de un alto funcionario, K.T. Li, y de Morris Chang, que llegó a la provincia china de Taiwán en 1985 por invitación del gobierno para hacerse cargo del ITRI y luego de la TSMC. ${ }^{20}$ Chang había sido vicepresidente de Texas Instruments y era el chino-americano más prestigioso en la industria estadounidense de alta tecnología.

Se establecieron en la provincia china de Taiwán tres compañías dedicadas al diseño de circuitos integrados (Quasel, Mosel y Vitelic), por iniciativa de "retornados" de los Estados Unidos (taiwaneses residentes en el extranjero que regresaron para vivir y trabajar en Taiwán) y con apoyo gubernamental. En 1985, las tres tenían dificultades financieras y nuevamente pidieron ayuda al gobierno. ${ }^{21}$ Querían que se instalara una fundición local especializada que pudiera proporcionarles un servicio más expedito y eficiente que el que les ofrecían los fabricantes extranjeros de dispositivos integrados que operaban en gran escala y que otorgaban baja prioridad a sus pedidos. Además, la propiedad intelectual de un diseño se protegía mejor con una fundición que con un fabricante de esos dispositivos.

La industria taiwanesa de diseño de circuitos integrados pasó de ocho casas en 1985 a 50 en 1988. Las ventas crecieron un $175 \%$ en 1988 y un $143 \%$ en 1989. Esto se debió en parte a la expansión del mercado interno y en parte al establecimiento de la TSMC. ${ }^{22}$ En 1999, el 91\% del trabajo de fabricación requerido por las casas de diseño de circuitos integrados se realizaba en el país. Las siete casas principales representaban, según una estimación, entre el $60 \%$ y el $70 \%$ de los ingresos totales por diseño de circuitos. En el mismo

\footnotetext{
${ }^{19}$ Las etapas que componen la producción de un circuito integrado son las siguientes: diseño, fabricación, recubrimiento (sellado), encapsulado y verificación.

${ }^{20}$ A la postre, la UMC adoptó la estrategia de la TSMC y redujo su integración vertical para asumir la estructura de una fundición. ${ }^{21}$ La ERSO inició en 1983, junto con el Consejo Nacional de Ciencias, un programa de fabricación de chips dividido en varios proyectos a fin de contribuir a ampliar la capacidad de diseño de circuitos integrados de las universidades taiwanesas (Chen y Sewell, 1996). Mosel y Vitelic se fusionaron en diciembre de 1991 y ahora fabrican y comercializan en todo el mundo chips de memoria dinámica con acceso aleatorio (DRAM) y otros productos. Sus ingresos llegaron a los 880 millones de dólares en 2000. Quasel ya no existe. 22 Véase Market Intelligence Center (1989, p. 390), Lin (1987), y Chang y Tsai (2000).
}

año, el $62 \%$ de la producción de semiconductores se vendió en el mercado local. ${ }^{23}$

Las compañías taiwanesas que se dedican al diseño de circuitos se beneficiaron de industrias locales de apoyo, más allá de las fundiciones. La industria de recubrimiento de circuitos integrados, al igual que la fundición, fue establecida por el gobierno. La ERSO les traspasó tecnología de dos compañías estadounidenses (UMR en 1977 y Electromask en 1980) y comenzó a suministrar servicios comerciales de recubrimiento a los productores locales. Se separó entonces la división responsable, para convertirse en la Taiwan Mask Corporation en 1989. ${ }^{24}$ Se calcula que el hecho de contar con un servicio local de recubrimiento les ahorra a las empresas locales veinte o más días en el ciclo completo de producción del circuito integrado (Lin, 1987).

Algunas empresas electrónicas estadounidenses se instalaron en la década de 1960 en la zona industrial de exportación ubicada en el sur, para desarrollar procesos de encapsulado, verificación y ensamblado (entre ellas, General Instrument, Motorola, Microchip y Texas Instruments). Paulatinamente, estas industrias fueron tomando carácter local: Motorola y Microchip, por ejemplo, vendieron su capacidad de encapsulado a empresas locales en 1999. En ese año, la capacidad de encapsulado de la provincia china de Taiwán se situaba en el primer lugar del mundo: el $99 \%$ de la demanda de encapsulado interna era atendido por empresas locales, y la mitad de los pedidos a los encapsuladores locales provenían de empresas también locales (ITRI, 2000a).

El personal ocupado por la industria de diseño de circuitos integrados, así como por la industria de estos circuitos en su conjunto, provenía en buena parte de la ERSO y de otras instituciones o programas gubernamentales y, en menor medida, del extranjero. La mayoría de las primeras casas de diseño de circuitos integrados, como Syntek (1982), Holtek (1983) y PTD (1986) eran filiales de la ERSO o habían sido establecidas por antiguos funcionarios de esta institución. A su vez, estas empresas tuvieron derivados propios (inesperados), como Chip Design Technology (1985) y Tontek (1986), que nacieron de Syntex. Cuando las empresas extranjeras de diseño, como Motorola y Philips, establecieron plantas de diseño de circuitos integrados en Taiwán a fines del decenio de 1980, contrataron personal de la

\footnotetext{
${ }^{23}$ Véase Ministerio de Finanzas, http://www.gio.gov.tw/taiwanwebsite/5-gp/eu/tables.htm.

24 Véase ERSO, 1994.
} 
ERSO o bien pidieron a ésta que se encargara de sus programas de capacitación (Lin, 1987). Los retornados sólo tuvieron cierta importancia a fines de la década de 1990. De las diez mejores casas taiwanesas de diseño de circuitos integrados, probablemente dos estaban a cargo de retornados en 1989, pero ya en 1995 ese número había aumentado a cinco (Hsu, 1997).

\section{V}

\section{Inicio de actividades: metas empresariales}

La provincia china de Taiwán fue una de las primeras economías de industrialización reciente en la que prosperaron las inversiones de capital de riesgo, que tuvieron un papel decisivo en el apoyo a la industria taiwanesa de alta tecnología. ${ }^{25} \mathrm{El}$ gobierno actuó como catalizador, ya que comenzó a fomentar los fondos de capital privado de riesgo para financiar empresas noveles en 1983, e incluso creó su propio fondo de capital de riesgo en la misma época. ${ }^{26}$

Sin embargo, no puede atribuirse al capital de riesgo el gran número de empresas noveles locales (aunque ese número esté descendiendo aceleradamente). Según los datos sobre el capital de riesgo para el período 1995-2000, las nuevas empresas recibían sólo una pequeña parte de los fondos. De las cinco etapas habituales del ciclo vital de una empresa (generación, inicio, expansión, madurez y reestructuración), el inicio de actividades mereció sólo $13,3 \%$ del total del

25 "El 70\% del capital de riesgo se ha dirigido a los sectores de computación y de electrónica. El resto se ha invertido en comunicaciones, productos industriales y artículos médicos y de biotecnología. La concentración del capital de riesgo taiwanés en las industrias tecnológicas más que en las tradicionales, que es similar a lo sucedido en Singapur y la República de Corea, puede atribuirse a los incentivos tributarios que canalizan ese tipo de capital hacia las inversiones en empresas tecnológicas (Wang, 1995, p.86). ${ }^{26}$ El Ministerio de Finanzas promulgó en 1983 un Reglamento sobre las empresas de inversiones de capital de riesgo, que establece las orientaciones para la organización de empresas de capital de riesgo, las exigencias mínimas de capital, y normas para la administración y supervisión de fondos. La primera compañía de capital de riesgo fue la Multiventure Capital Corporation (1984), proyecto conjunto de Acer y Continental Construction. Luego se estableció una empresa mixta entre una compañía estadounidense de capital de riesgo (Hambrest \& Quest) y varias dependencias del gobierno, en la que un $49 \%$ del capital mínimo exigido a las empresas provenía del fondo gubernamental de desarrollo (el Yuan Ejecutivo), y un cuasi banco de desarrollo (el Chiao-Tung). Véase Tzeng (1991) y Taiwan Venture Capital Association (http://www.tvca.org.tw/indexe.htm). financiamiento con capital de riesgo en 1995. ${ }^{27}$ Suponiendo que las estadísticas disponibles son correctas, la parte principal del financiamiento se dedicó a los períodos de expansión y madurez, lo cual incluye la transformación de compañías privadas en compañías públicas mediante ofertas públicas iniciales. ${ }^{28} \mathrm{Hacia}$ el año 2000 la participación del total del capital de riesgo destinado a las empresas noveles había aumentado a $32,8 \%$, proporción todavía inferior a la dedicada a las etapas de expansión y madurez.

En la mayoría de los casos en que un agente externo incubaba una empresa que iniciaba sus actividades, ese agente era el gobierno y no un socio capitalista privado. A veces el gobierno impulsaba directamente ese inicio, como ocurrió con la creación de las dos fundiciones de semiconductores de propiedad estatal y de nivel internacional, UMC y TSMC. Sin embargo, era habitual que el gobierno promoviera el inicio de actividades de forma indirecta mediante el financiamiento, las instalaciones y el acceso a tecnologías indispensables para su crecimiento.

La creación de parques científicos fue un método muy importante para fomentar el nacimiento de industrias; el primero se emplazó en el sur de Taipei, en Hsinchu, y el segundo al sur de la isla de Taiwán, en Tainan. El gobierno seleccionaba una a una las

\footnotetext{
${ }^{27}$ El financiamiento del inicio de actividades puede haber sido más importante en la década de 1980 que en la de 1990. Se calcula que de unas 80 empresas que operaban en el Parque científico-industrial de Hsinchu en 1987, un total de 43 estaban financiadas con capital de riesgo (Liu y otros, 1989).

28 'Los logros de las inversiones de capital de riesgo en la labor de mostrar a las empresas tecnológicas cómo abrir su oferta de acciones al público son considerables' (Wang, 1995, p.90). Las ofertas públicas fueron la principal estrategia de retirada de los inversionistas de capital de riesgo.
} 
empresas que podían acceder a estos parques. Los residentes de los parques recibían un conjunto completo de generosos y amplios subsidios que incluían exenciones de impuestos y derechos de importación, donaciones y créditos subvencionados, naves industriales o emplazamientos de alta calidad con precios de alquiler inferiores a los del mercado, ayudas para el día a día de los investigadores de alto rango (entre las que se incluía la enseñanza bilingüe para los hijos de expatriados) y acceso a los servicios gubernamentales y universitarios de investigación. 'El motor del crecimiento económico taiwanés en la década de 1980 fue la industria de la informática, y el maquinista fue el parque científico-industrial' (Liu y otros, 1989, p.35). ${ }^{29}$ Del gasto total del gobierno en actividades de investigación y desarrollo, se destinaba al Parque científico-industrial de Hsinchu una proporción considerable y creciente (llegó hasta el 18\% en 1998), aunque este generaba menos del $1 \%$ de la producción total, medido por las ventas como proporción del producto nacional bruto. Por último, mientras el número de licenciados en ciencias de las universidades crecía de 6.000 en 1990 a 16.000 en 1998, la proporción de estos titulados que encontraban empleo en las empresas del parque Hsinchu pasaba del $2 \%$ a más del $6 \%$ en el mismo período.

\section{VI}

\section{Estándares de desempeño}

Como se ha visto, pese a la adhesión declarada del gobierno taiwanés a los principios de la liberalización, y pese a haber abierto sus mercados a una mayor competencia extranjera, en la práctica su política industrial siguió teniendo un papel importante en el sector de alta tecnología del país. El éxito general de la política industrial (evaluada por su participación en el mercado mundial de tecnología de la información) se reflejaba en la madurez tecnológica que iba adquiriendo la economía taiwanesa y en sus estándares de desempeño.

Los productos de alta tecnología en los que el gobierno centró su estrategia de sustitución de importaciones ya eran productos maduros según los cánones mundiales, es decir, sus ventas habían alcanzado un alto volumen antes de que comenzara su producción en la provincia china de Taiwán. Siendo así, el riesgo del gobierno era limitado: las empresas nacionales tenían que hacer frente a la incertidumbre económica pero no se adentraban en un territorio tecnológico desconocido. Sin embargo, el margen potencial de error a que se exponía el gobierno era bastante grande, porque la inseguridad tecnológica no era necesariamente insignificante, a pesar de la madurez de los productos. Por ejemplo, el gobierno acertó, entre varias posibilidades, en el caso de la tecnología de la informática. Su decisión de 'apostar por los semiconductores complementarios de óxido metálico (CMOS) fue decisiva para que Taiwán pudiera sincronizar el desarrollo de la tecnología de los semiconductores con su tecnología de la informática basada en las computadoras personales a fin de lograr el más alto efecto sinérgico' (Chang y Tsai, 2000, p.187). ${ }^{30}$ La selección del gobierno se basaba en estudios detallados y concertados sobre los avances y las tendencias tecnológicas a cargo de comités formados por funcionarios de gobierno, empresarios y expertos universitarios. Además, la distribución de los subsidios resultó positiva en general, porque, como en el pasado, el gobierno la supeditó a estándares concretos de rendimiento, cuantificables y comprobables. ${ }^{31}$ Lo diferente al alcanzar la etapa de alta tecnología era que estos estándares tendían a incrementar las inversiones en activos basados en los conocimientos.

Los estándares de desempeño fueron útiles para las industrias taiwanesas de alta tecnología en dos aspectos: como criterios que tenían que cumplir las empresas para optar a los subsidios gubernamentales y como condición para seguir recibiendo incentivos. El gobierno debía ser selectivo, ya que la demanda de subsidios por parte de las empresas y los institutos de investigación superaba la oferta. La condicionalidad resultaba eficaz porque el sector manufacturero taiwanés — según testimonio de las empresas entrevistadas-

\footnotetext{
${ }^{29}$ Véanse también Chang (1992); Yang (1998); y Hsinchu ScienceBased Industrial Park (2000), publicación No. 2309. 30 En cuanto a esta decisión, véase Chang y otros (1994). ${ }^{31}$ En Amsden (2001) puede verse un análisis general de las normas de eficiencia para el éxito de la intervención estatal en el proceso de industrialización.
} 
había acumulado experiencia y competencia suficientes como para fabricar redituablemente productos de alta tecnología. A medida que los proyectos se volvían rentables, generaban el ingreso necesario para que los beneficiarios pudieran reembolsar sus préstamos y cumplir las exigencias de investigación y desarrollo impuestas por el gobierno, que eran la condición principal del programa. Los proyectos que tenían éxito reforzaban a su vez las campañas de promoción gubernamentales.

Las condiciones de admisión al Parque científicotecnológico de Hsinchu (hacia 1980) eran las siguientes:

i) la empresa debía ser capaz de diseñar productos para su fabricación según un plan estratégico empresarial;

ii) debía fabricar productos cuyo proceso de investigación y desarrollo todavía estuviera en evolución;

iii) tenía que haber fabricado productos con potencial de desarrollo e innovación;

iv) debía tener experiencia en innovación y en investigación y desarrollo de alto nivel en un departamento de investigación que cumpliera ciertas exigencias mínimas de tamaño;

v) debía haber aplicado procesos de fabricación que requirieran capacitación en técnicas avanzadas, o bien inversiones considerables en actividades de investigación y desarrollo;

vi) después de tres años de comercializar un producto o un servicio, al menos el $50 \%$ del personal debería estar compuesto por técnicos de origen local, y

vii) sus operaciones debían contribuir significativamente a la reconstrucción económica de la provincia china de Taiwán y a la defensa (Liu y otros, 1989).

Los ganadores de los concursos eran elegidos por un comité tripartito de expertos provenientes de la industria privada, el gobierno y el ámbito académico, como ocurría con otros programas gubernamentales.

Para acceder a los fondos fiscales destinados a productos o industrias de carácter estratégico (como el CD-ROM o las pantallas de TFT) había que cumplir las normas siguientes: i) las empresas debían acreditar su solvencia financiera y su capacidad económica;

ii) debían demostrar que tenían un departamento de investigación en funcionamiento, $y$

iii) debían documentar logros sustanciales en materia de investigación y desarrollo.

Una vez que se desarrollaba con éxito un producto que disfrutaba de promoción fiscal, los derechos de propiedad intelectual se asignaban de la siguiente manera:

i) la propiedad se compartía a partes iguales, en copropiedad, por el Ministerio de Economía y la empresa que había desarrollado el producto, ya que el gobierno había cubierto el $50 \%$ de los costos totales de desarrollo;

ii) si el Ministerio deseaba vender una parte de sus derechos de propiedad intelectual, la empresa que los compartía tenía el derecho de opción prioritaria, y

iii) si la empresa no llegara a iniciar la producción o la venta del producto principal programado a los tres años de finalizado el plan de desarrollo (por motivos como quiebra, estrategia de comercialización o dificultades de operación), perdería no sólo los derechos de propiedad intelectual sino que tendría que restituir al gobierno en cuotas, el dinero invertido.

Para recibir subsidios de investigación y desarrollo, las empresas de los sectores estratégicos tenían que comprometerse a gastar cierta proporción de sus propios recursos en nuevas actividades de investigación y desarrollo. Esa proporción dependía en parte del tamaño de la empresa. Mientras más grande fuera, mayor sería la proporción. Si la cantidad gastada fuera inferior a lo estipulado, la empresa tendría que aportar el faltante a un fondo de investigación y desarrollo señalado por el gobierno.

En general, el gobierno fomentaba activamente la acumulación en las industrias estratégicas de activos vinculados, a fin de mantener el prestigio de la provincia china de Taiwán como base de producción. Los estándares de desempeño que exigía para otorgar subsidios contribuía a impulsar la obtención de un alto valor agregado. 


\section{VII}

\section{La industria de alta tecnología en América Latina}

La industria de alta tecnología se caracteriza por su uso más tácito que explícito de la tecnología, merced a su capacidad específica y exclusiva de crear productos nuevos y obtener una rentabilidad superior a la normal. Por lo tanto, las industrias de alta tecnología son convenientes para los países porque requieren trabajadores calificados bien pagados, y ofrecen a los empresarios la posibilidad de obtener utilidades tecnológicas. Por definición, la rentabilidad de un producto de alta tecnología sigue siendo superior a la normal para una economía de industrialización reciente, aun cuando la tecnología involucrada sea madura según los estándares de los países desarrollados.

El predominio de los Estados Unidos y de Asia en la electrónica hace muy difícil a los demás países penetrar en este campo. Asia no sólo fabrica a bajo costo casi todos los productos electrónicos maduros, gracias a sus bien desarrolladas empresas privadas y sus sistemas de innovación locales, sino que también aprovecha externalidades tanto nacionales como regionales (Fujita y otros, 2000).

América Latina tiene dos opciones para crear un sector de alta tecnología y, por ende, las empresas y especialistas necesarios para que ese sector prospere. En primer lugar, puede alentar a las empresas de propiedad extranjera que dominan algunos sectores, como el automovilístico, a que incrementen sus actividades locales de investigación y desarrollo. Como se aprecia en el cuadro 1, la alta tecnología, definida en términos amplios, incluye sectores que no hacen uso especialmente intensivo de las actividades de investigación y desarrollo, como el de equipos de transporte, junto a otros que sí lo hacen, como las industrias electrónica y química. Sin embargo, los principales productores de automóviles, como Volkswagen y General Motors, cuentan con grandes laboratorios de su propiedad en sus países de origen. Por un lado, estas empresas se ven incentivadas a trasladar las actividades de investigación y desarrollo a países menos desarrollados, por sus bajos costos de ingeniería. Pero por otro lado, las desincentiva la escasez de especialistas con experiencia, la inseguridad sobre los derechos de propiedad intelectual y la renuencia de los científicos de los laboratorios centrales a ceder el control de los proyectos de investigación más interesantes. Los altos ejecutivos de tales empresas quieren mantener estos proyectos en su lugar de origen y bajo su supervisión.

El gobierno de Singapur logró vencer estas reticencias de las empresas extranjeras, que representan cerca del $80 \%$ del valor agregado total en la manufactura del país, mediante la aplicación sistemática de un esquema de incentivos y la creación de un conjunto de instituciones encargadas de inducir a estas empresas a realizar localmente más labores de investigación y desarrollo (Amsden y otros, 2001). De hecho, los proyectos más avanzados y prometedores de investigación y desarrollo, en términos de capacitación de especialistas y de desarrollo de nuevos productos, se realizaron en laboratorios de propiedad del gobierno, que actuaban de forma independiente. Esos mismos laboratorios también cooperaban con las empresas extranjeras en la solución de sus problemas de producción y, más tarde, en cuestiones relacionadas con investigaciones avanzadas. Además, el gobierno protegía escrupulosamente la propiedad intelectual y subsidiaba sistemáticamente las actividades de investigación y capacitación del sector privado. Esta actitud contribuyó a incrementar las iniciativas de investigación y desarrollo de las empresas extranjeras en Singapur. Un escenario similar podría plantearse en América Latina.

En segundo lugar, América Latina se ha distinguido por la producción de bienes petroquímicos. Algunos países, sobre todo Brasil y en cierta época Argentina, también descollaban en la producción farmacéutica. Estas dos industrias, combinadas con la biotecnología, abarcan un amplio sector con diversas oportunidades para fabricar nuevos productos de alta tecnología.

Cualquiera sea su potencial, el total de actividades de investigación y desarrollo industrial en América Latina representa una proporción mucho más baja del producto nacional bruto que en Asia (cuadro 4). Hay muy pocas empresas latinoamericanas activas en este campo, incluso en Brasil (Alcorta y Peres, 1998). El sistema educativo no se orienta a la investigación que tiene aplicaciones industriales. Los laboratorios gubernamentales también han sido descuidados por el 
afán liberal de recortar el presupuesto público. Por lo tanto, América Latina tiene ante sí una ingente tarea de formulación de políticas y desarrollo de instituciones si pretende promover la industria de alta tecnología. El problema no radica en las normas de la OMC (que en general tampoco ayudan mucho), sino en las inadecuadas instituciones vinculadas con la tecnología que surgieron como resultado del Consenso de Washington.

CUADRO 4

\section{Gasto en investigación y desarrollo, 1985 y 1995 \\ (Porcentaje del producto nacional bruto)}

\begin{tabular}{lcc}
\hline & $1985^{\mathrm{a}}$ & $1995^{\mathrm{b}}$ \\
\hline República de Corea & 1,8 & 2,8 \\
Provincia china de Taiwán & 1,2 & 1,8 \\
India & 0,9 & 0,8 \\
Chile & 0,5 & 0,7 \\
Brasil & 0,7 & 0,6 \\
Turquía & 0,6 & 0,6 \\
China & $\ldots$, & 0,5 \\
Argentina & 0,4 & 0,4 \\
Malasia & $\ldots$, & 0,4 \\
Indonesia & 0,3 & 0,1 \\
Tailandia & 0,3 & 0,1 \\
México & 0,2 & 0,0 \\
\hline
\end{tabular}

Fuente: Para la provincia china de Taiwán: Taiwan National Science Council (1996). Para lo demás, Unesco (varios años).

a Los datos sobre India e Indonesia son de 1986; sobre Brasil, México, la República de Corea y Turquía, de 1987; sobre Chile y la provincia china de Taiwán, de 1988.

b Los datos sobre Malasia son de 1992; sobre México, de 1993; sobre India y la República de Corea, de 1994.
La creación de instituciones para fomentar la alta tecnología fue un elemento crucial del éxito taiwanés, como se aprecia en el caso de las industrias de diseño de CD-ROM, pantallas TFT y circuitos integrados. Esas instituciones, que exigían una actuación protagónica pero disciplinada por parte del gobierno, tenían los rasgos de aquellas que inspiraron los escritos de Raúl Prebisch sobre el desarrollo económico. Por lo tanto, puede afirmarse que Prebisch ha renacido en Asia.

Está claro que la mayor parte de las inversiones privadas o públicas en nuevas tecnologías terminan por fracasar. Sin embargo, si los países no invierten en tecnología, sus economías seguramente fracasarán. ¿Abrirá América Latina sus puertas nuevamente a políticas neo- prebischianas, pero esta vez para crear industrias de alta tecnología?

(Traducido del inglés)
Alcorta, L. y W. Peres (1998): Innovation systems and technological specialization in Latin America and the Caribbean, Research Policy, No 26, Amsterdam, Elsevier Science.

Amsden, A.H. (1989): Asia's Next Giant: South Korea and Late Industrialization, Nueva York, Oxford University Press. (2001), The Rise of 'the Rest': Challenges to the West from Late-Industrializing Economies, Nueva York, Oxford University Press.

Amsden, A.H. y otros (2001): Do foreign companies conduct R\&D in developing countries?, A New Approach to Analyzing the Level of $R \& D$ with an Analysis of Singapore, Tokio, Instituto del Banco Asiático de Desarrollo.

Amsden, A.H. y T. Hikino (1994): Project execution capability, organizational know-how and conglomerate corporate growth in late industrialization, Industrial and Corporate Change, vol. 3, $\mathrm{N}^{\mathrm{o}} 1$, Oxford, Oxford University Press.

Amsden, A.H. y W.W. Chu (2003): Beyond Late Development: Taiwan's Upgrading Policies, Cambridge, Massachusetts, MIT Press.

Cochran, T. y R. Reina (1962): Entrepreneurship in Argentine Culture, Filadelfia, University of Pennsylvania Press.

Chang, C.C. (1992): The Development of Taiwan's Personal Computer Industry. Taiwan's Enterprises in Global Perspective, Nueva York.
Chang, P.L. y C.T. Tsai (2000): Evolution of technology development strategies for Taiwan's semiconductor industry: formation of research consortia, Industry and Innovation, vol. 7, $\mathrm{N}^{\mathrm{o}} 2$, Londres, Taylor \& Francis Group.

Chang, P.L. y otros (1994): The formation process of Taiwan's IC industry. Method of technology transfer, Technovation, vol. $14, \mathrm{~N}^{\mathrm{o}} 3$, Amsterdam, Elsevier Science.

Chen, C.F. y G. Sewell (1996): Strategies for technological development in South Korea and Taiwan: the case of semiconductors, Research Policy, $\mathrm{N}^{\circ}$ 25, Amsterdam, Elsevier Science.

ERSO (Electronics Research and Service Organization) (1994): ERSO: The First Twenty Years, Hsinchu, Taipei.

Evans, P. (1995): Embedded Autonomy: States and Industrial Transformation, Princeton, Princeton University Press.

Fields, K.J. (1995): Enterprise and the State in Korea and Taiwan, Ithaca, Cornell University Press.

Fujita, M. y otros (2000): The Spatial Economy: Cities, Regions, and International Trade, Cambridge, Massachusetts, мIт Press.

Hsiao, F.H. (1994): Taiwan's Industrial Policy and Industrial Development, Taipei, Far East Economic Research.

Hsinchu Science-Based Industrial Park (2000): Publicación Nº 2309, Hsinchu.

Hsu, J.Y. (1997): An historical study of Taiwan's IC industry, Journal 
of Geography Science, $N^{\circ}$ 23, Taipei, National Taiwan University.

Industrial Development Bureau (varios años): Industrial Development Yearbook, Taipei.

ITIS (Industrial Technology Information Service) (1989): Annual Report, 1998 [http://www.itri.org.tw/eng/about/annual/ annual98/spec_pg3.jsp].

ITRI (Industrial Technology Research Institute) (1997): Summary Report of the Key Components and Products Project, Taipei. (1999): Taiwan's Prospects for Developing the TFTLCD Industry, Taipei.

(2000a): A Study of Taiwan's Semiconductor Companies, Taipei, Industrial Technology Research Institute

(ITRI)/Industrial Economics and Knowledge Center (IEK) [http://www.itri.org.tw/eng/about/annual/annua198 /spec pg3.jsp].

(2000b): Study on the Development Trend of the Flat Panel Display Industry, Taipei [http://www.itri.org.tw/eng/about /annual/annual98/spec_pg3.jsp].

Lin, H.M. (1987): A study of an emerging industry in a LDC: A case study of Taiwan's IC industry, Economics, Taipei, National Taiwan University.

Lindblad, J.T. (comp.) (1996): Historical Foundations of a National Economy in Indonesia, 1890s-1990s, Amsterdam, NorthHolland.

Linden, G. y otros (1998): Flying geese as moving targets: are Korea and Taiwan catching up with Japan in advanced displays?, Industry and Innovation, vol. 5, $\mathrm{N}^{\circ} 1$, Londres, Taylor \& Francis Group.

Liu, P.K.C. y otros (1989): New technologies, industry and trade: the Taiwan experience, Industry of Free China, Taipei, noviembre.

Mallon, R.D. y W.J.V. Sourrouille (1975): Economic Policymaking in a Conflict Society: The Argentine Case, Cambridge, Massachusetts, Harvard University Press.

Market Intelligence Center (1989): IT Industry Yearbook, 1989, Taipei, Institute for Information Industry.

(varios años): IT Industry Yearbook, Taipei, Institute for Information Industry.

Nikkei Weekly (2001): IBM, Toshiba calling halt to LCD output, Tokio.

San, G. (1995): An Overview of Policy Priorities for Industrial Development in Taiwan, Taipei, National Central University.

Sridharan, E. (1996): The Political Economy of Industrial Promotion: Indian, Brazilian, and Korean Electronics in Comparative Perspective, 1969-1994, Westport, Praeger.

Tomlinson, B.R. (1981): Colonial firms and the decline of colonialism in Eastern India, 1914-47, Modern Asian Studies, vol. 15, No 3, Nueva York, Cambridge University Press.

Tzeng, Y.C. (1991): Leaders of Taiwan's venture capital industry, Wealth Magazine, junio.

Wang, L.R. (1995): Taiwan's venture capital: policies and impacts, Journal of Industry Studies, vol. 2, No 1, Londres, Taylor \& Francis Group.

Wong, P.K. y J.A. Matthews (1998): Competing in the global flat panel display industry: introduction, Industry and Innovation, vol. $5, \mathrm{~N}^{\circ} 1$, Londres, Taylor \& Francis Group.

Yang, Y.R. (1998): The political economy of a science park, Economics, Taipei, National Taiwan University. 